\title{
Shape and Position of the Sarcoplasmic Reticulum and the Golgi Apparatus in the Sole Plate and Remaining Subsarcolemmal Muscle Region of the Mouse Using Imidazole-Osmium Staining
}

\author{
TILMAN VOIGT, ${ }^{1 *}$ WOLFGANG DAUBER, ${ }^{2}$ IRINA BENSEMANN-RYVKIN,${ }^{2}$ AND XENIA HÄRTEL ${ }^{2}$ \\ ${ }^{1}$ Institute of Anatomy, University of Fribourg, $\mathrm{CH}-1700$ Fribourg, Switzerland \\ ${ }^{2}$ Institute of Anatomy, Eberhard-Karls-University Tuebingen, 72074 Tuebingen, Germany
}

\begin{abstract}
KEYWORDS morphology; neuromuscular junction; skeletal muscle fiber; ultrastructure
\end{abstract}
\begin{abstract}
By means of thin $(\leq 150 \mathrm{~nm})$ and thick $(>150 \mathrm{~nm})$ sections, the shape and position of the sarcoplasmic reticulum and of the Golgi apparatus in the sole plate and in the remaining subsarcolemmal sarcoplasmic region were investigated. For this purpose the membranes were stained by means of imidazole-osmium postfixation and unstained sections analyzed under the electron microscope. Both in the sarcoplasma of the sole plate and around the muscle fiber nuclei, a network of tubules is visible after imidazole-osmium staining which can be identified as the sarcoplasmic reticulum solely on the basis of its contacts with the perinuclear cistern and the cisterns of the triads. Findings in literature on the position of the Golgi apparatus are confirmed and similar spatial relationships and vesiculations between the perinuclear cisterns and the Golgi apparatus of the sole plate nuclei and the other subsarcolemmal fiber nuclei are also demonstrated using this new staining method.
\end{abstract}

\section{INTRODUCTION}

Earlier investigations of the motor end plate (MEP) have shown that the transversal tubular system equals the T-system, which is responsible for the excitation propagation into the interior of muscle fibers. This system has a net-like architecture in the sole plate with continuous connections to the subsynaptic folds (Dauber et al., 1999, 2000). The sarcoplasmatic reticulum (SR) also occurs in the MEP in a rough and smooth form. Extensive information is available about the shape, extent, and molecular equipment and the proteins of the myofibrillar smooth SR of muscle fibers which run along the myofibrils and the subsarcolemmal space outside the MEP (Volpe et al., 1992; Villa et al., 1993). However, there is a lack of corresponding information about the SR in the sole plate. In histological sections T-tubules and tubules of the smooth SR cannot be distinguished without the existence of special structure characteristics like triads, which are a combination of two SR cisterns with a central T-tubules (Dauber et al., 1999), or without the filling of the T-system with extracellular markers; for example, lanthanum (Dauber et al., 2000).

The aim of the present examination was to analyze more exactly the shape of the SR in the sole plate. For this purpose sole plates of muscle fibers of mice diaphragms were examined with the aid of imidazoleosmium postfixation, which is especially suitable for the representation of membranes and, furthermore, makes the conventional staining of sections to be examined unnecessary (Voigt et al., 2002). The SR was identified due to its connections with the perinuclear cistern and through the coformation of triads. In order to raise the likelihood of a successful search, an exam- ination was carried out also on thick sections $(>150 \mathrm{~nm})$. Additionally, it turned out that the method is suitable for visualizing the relation between the Golgi apparatus and the perinuclear cisterns, of both the sole plate nuclei and the fiber nuclei outside of the MEP.

\section{MATERIALS AND METHODS}

Mice $(\mathrm{n}=3)$ were perfused under anesthetic (Rompun-Ketanest) with $2.5 \%$ glutaraldehyde in $0.1 \mathrm{M}$ cacodylate buffer. The middle third of the diaphragm (Bowden and Duchen, 1976) (which is especially rich in end plates) was prepared and cut into small blocks in a Petri dish filled with the same fixating agent. The blocks were left for a further hour in the same fixatives (Dauber et al., 1999, 2000). Then the tissue was postfixed for 1 hour at $4^{\circ} \mathrm{C}$ in $1 \%$ osmium with $0.1 \mathrm{M}$ imidazole in $0.1 \mathrm{M}$ cacodylate buffer ( $\mathrm{pH}$ 7.2). After postfixation, the blocks were soaked $3 \times 5 \mathrm{~min}$ in distilled water, dehydrated by an ascending alcohol series, and embedded in Epon. The sections were mounted on slot apertures to prevent covering of motor end plates by grid crosspieces. The film stability was increased through supplement of $25 \mu$ l dibutyl phthalate (Kneissler, 1997) onto $50 \mathrm{ml} 0.5 \%$ Pioloform. Unstained thin $(\leq 150 \mathrm{~nm})$ and thick $(>150 \mathrm{~nm})$ sections

\footnotetext{
*Correspondence to: Tilman Voigt, Institute of Anatomy, University of Fribourg, Rte Albert-Gockel 1, CH-1700 Fribourg, Switzerland.

E-mail: tilman.voigt-gawatz@unifr.ch

Received 18 October 2002; accepted in revised form 30 December 2002 DOI 10.1002/jemt.10308
} 


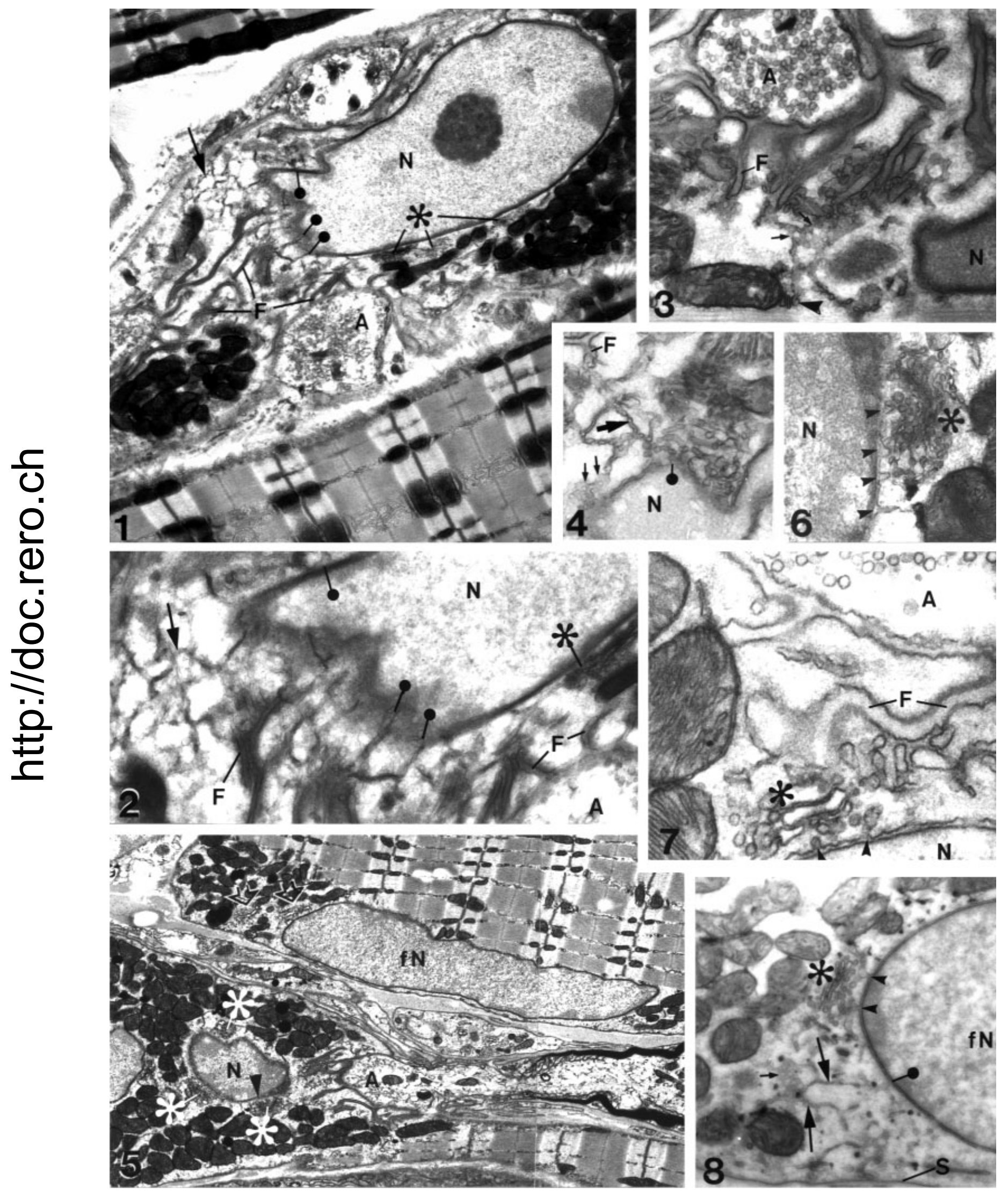

Figs. 1-8 
were examined in a transmission electron microscope (LE0912 Omega; Zeiss).

\section{RESULTS}

This investigation concentrated on the sarcoplasma of the sole plate and the sarcoplasma in the vicinity of the nuclei outside of the MEP. A distinctly structured tubular network was found in both sarcoplasmic areas outside of their accumulation of mitochondria. As expected, in thin and thick sections it was possible to view a different large portion of this network. This network features distinct connections to the perinuclear cistern of a sole plate nucleus (Figs. 1, 2, 4) (see also fig. 3 in Voigt et al., 2002), the SR cistern of a triad (Fig. 3) and the perinuclear cistern of a muscle fiber nucleus (Fig. 8). Because of the shown contacts, this network can be viewed as belonging to the SR. Morphologically, it is possible to differentiate between two portions of this net: a wide-lumened, closely knit portion with round clearings (Figs. 3, 4, 8) and a narrowlumened, loosely knit portion (Figs. 1, 2, 4). All in all, the appearance of the wide-lumened, closely knit portion is reminiscent of a more slender version of the fenestrated collar near the $\mathrm{M}$ band of a myofibril.

A Golgi apparatus is regularly present in the sole plate, usually distributed in portions in close proximity to the perinuclear cistern of the sole plate nucleus (Figs. 2, 5). However, the Golgi apparatus has a similar relationship to the subsarcolemmal nuclei of the muscle fiber far outside the MEP (Fig. 5). Near the Golgi apparatus, the perinuclear cistern displays vesicle-like protrusions pointing toward it (Fig. 6). Protrusions of this kind appear to be more numerous at the sole plate nuclei than at the fiber nuclei far outside the MEP. Because the sole plate nuclei are located just below the subsynaptic folds, there are also close spatial relationships between the sarcolemma of the folds and the Golgi apparatus (Fig. 7).

\section{DISCUSSION}

The investigation has shown that imidazole-osmium postfixation without conventional section staining is effective in displaying the morphology of membrane structures. Thus, a tubular network underneath the subsynaptic folds is shown here which, when imidazole-osmium postfixation is used (as opposed to the $\mathrm{Ur}-\mathrm{Cu}-\mathrm{Pb}$ technique [Segretain, 1995]), is revealed to be a membrane-covered tubular system. Only on the basis of its contacts with the perinuclear cistern or the cistern of the triads is it possible to recognize this tubular network as belonging to the SR.

The use of conventional section-staining after the imidazole-osmium postfixation for the purpose of highlighting the ribosomes can serve to differentiate the rough SR of the sole plate sarcoplasm (Voigt et al., 2002). However, it is still not possible to differentiate between profiles of the smooth SR of the sole plate and T-tubules of the sole plate (Dauber et al., 2000). Here the same problems of visualizing and differentiation between the myofibrillar smooth SR and the myofibrillar longitudinal T-system occur in the area of the myofibrils (Peachey, 1965; Eisenberg et al., 1974; Dulhunty et al., 1984). The Ur-Cu-Pb method is likewise unsuitable for demonstrating the differences in structure. This method stains not only the SR, but also the myofibrillar T-system between the triads (see fig. 2 in Rambourg and Segretain, 1980). The Ur-Cu-Pb method causes confusion in distinguishing SR and T-tubular membranes in the sole plate. At present, there is no means of simultaneously highlighting both the SR system and the T-system of the sole plate so as to be able to completely map them in an ultrastructurally differentiated fashion. Thus, even ultrathin serial sections are unsuitable since in every section the issue would have to be set up for the identity of a tubular profile. In thick sections $(>150 \mathrm{~nm})$ more extensive portions of
Fig. 1. Section of a MEP in the area of the sole plate. The sarcoplasm of the sole plate is invested with a branching system of tubules $(\rightarrow)$. Exits of this tubular system from the perinuclear cistern $(-)$ can serve to identify them as sarcoplasmic reticulum; Golgi apparatus (*); presynapse (A), subsynaptic folds (F); sole plate nucleus $(\mathrm{N}) ; 300 \mathrm{~nm}$ thick section; 8,500:1.

Fig. 2. Detail of Figure 1. The sarcoplasm of the sole plate is invested with a branching system of tubules $(\rightarrow)$. Exits of this tubular system from the perinuclear cistern $(0)$ can serve to identify them as sarcoplasmic reticulum; Golgi apparatus (*); presynapse (A), subsynaptic folds (F); sole plate nucleus (N); $300 \mathrm{~nm}$ thick section; 20,000:1.

Fig. 3 Section of an MEP in the area of the sole plate. Beginning from the SR cistern ( $>$ ) of a triad, a tubular network extends to beneath the subsynaptic folds (F). There, the net thickens so that only small sarcoplasmic lumina $(\rightarrow)$ are visible between the individual tubules; presynapse (A), sole plate nucleus (N). $120 \mathrm{~nm}$ thick section; $25,000 \times$.

Fig. 4. Section of a sole plate directly beneath a sole plate nucleus. A loosely knit net is spread out $\rightarrow$ ) between the subsynaptic folds (F) and the perinuclear cistern. Near the cell nucleus, this loosely knit net transitions into a closely knit net with small holes $(\rightarrow)$. Transition between the net and the perinuclear cistern $(\mathbf{O})$; sole plate nucleus $(\mathrm{N}) ; 120 \mathrm{~nm}$ thick section; 25,000x.

Fig. 5. Longitudinal section through the mouse diaphragma with details of two muscle fibers. One muscle fiber shows the section through a motor end plate. On the subneural side of the sole plate nucleus $(\mathrm{N})$, three profiles of the Golgi apparatus $(*)$ are located just beneath the perinuclear cistern. From the presynapse (A) subsynaptic folds extend up to the Golgi apparatus. Region of detail in Figure 6 ( ). The other muscle fiber shows a fiber segment outside the MEP. Here profiles of the Golgi apparatus $(\Leftrightarrow)$ are found also at the end of the oblong muscle fiber nucleus (fN); $200 \mathrm{~nm}$ thick section; 5,000×.

Fig. 6. Detail of Figure 5. On the subneural side of the sole plate nucleus $(\mathrm{N})$, a Golgi apparatus (*) is located just beneath the perinuclear cistern. The perinuclear cistern features numerous protrusions (>) pointing towards it; $200 \mathrm{~nm}$ thick section; $25,000 \times$.

Fig. 7. Section of a sole plate. The Golgi apparatus (*) lies directly underneath the perinuclear cistern, where it features protrusions $(>)$ pointing towards the Golgi apparatus. At the same time, a subsynaptic fold $(\mathrm{F})$ extends up to the immediate vicinity of the Golgi apparatus; presynapse (A), sole plate nucleus (N); $120 \mathrm{~nm}$ thick section; $40,000 \times$

Fig. 8. Section of the sarcoplasma at the pole of a muscle fiber nucleus (fN). The Golgi apparatus (*) lies directly underneath the perinuclear cistern. Protrusions $(>)$ of the perinuclear cistern extend into the area of the Golgi apparatus. At the same time, a network of tubules $(\rightarrow)$ is visible which, due to its contact with the perinuclear cistern (-), can be identified as the SR. The closely knit portion of the network is so densely structured that only small sarcoplasmic lumens are visible through the mesh $(\rightarrow)$. Sarcolemma (S). $120 \mathrm{~nm}$ thick section; $25,000 \times$ 
the tubular network of the smooth SR can be shown than is possible in thin sections (Dauber et al., 2000).

Portions of the network resemble the fenestrated collar around the myofibrils, which is responsible for the return transport of released $\mathrm{Ca}^{2+}$. In analogy to this function, one could postulate that a certain $\mathrm{Ca}^{2+}$ environment in the sole plate is being maintained by means of the return transport of $\mathrm{Ca}^{2+}$ which is released in the sarcoplasm of the sole plate by the triads of the sole plate (Dauber et al., 1999, 2000) and which streams into the sarcoplasm of the sole plate through the subsynaptic folds (Decker and Dani, 1990).

There is a controversial discussion concerning the position of the Golgi apparatus in adult skeletal muscle tissue. Thus, the Golgi apparatus in adult skeletal muscle tissue is supposedly located only in the sole plate and is associated with the subneural sole plate nuclei (Jasmin et al., 1989, 1990, 1995; Cartaud and Changeux, 1993; Cartaud et al., 2000). However, in adult skeletal muscle tissue it has also been shown outside of the sole plate at the poles of the muscle fiber nuclei (Ralston, 1993). As demonstrated here, the Golgi apparatus is located both in the sarcoplasma of the sole plate and around the remaining muscle fiber nuclei outside the MEP. Furthermore, in both locations there are comparable shapes of protrusions from the perinuclear cistern to the Golgi apparatus. Thus, we can recognize as in myotubes not only a contiguity between the Golgi apparatus and the perinuclear cistern (Tassin et al., 1985), but also an increased number of protrusions of the perinuclear cistern in direction of the Golgi apparatus without microtubules between these two structures. Because of this contiguity in myotubes, geometrical difficulties in the transport of vesicles between the rough and smooth SR and the cis-side of the Golgi apparatus are discussed (Tassin et al., 1985). Since the SR touches the perinuclear cistern, as shown here, a transport of substrate by means of protrusions in the direction of the Golgi apparatus should be possible.

\section{REFERENCES}

Bowden RRM, Duchen LW. 1976. The anatomy and pathology of the neuromuscular junction. In: Zaimis E, Maclagan J, editors. Handbook of experimental pharmacology vol. 42. Neuromuscular junction. Berlin: Springer-Verlag, p 23-97.

Cartaud J, Changeux JP. 1993. Post-transcriptional compartmentalization of acetylcholine receptor biosynthesis in the subneural domain of muscle and electrocyte junctions. Eur J Neurosci 5:191202
Cartaud J, Cartaud A, Kordeli E, Ludosky MA, Marchand S, Stetzkowski-Marden F. 2000. The torpedo electrocyte: a model system to study membrane-cytoskeleton interactions at the postsynaptic membrane. Microsc Res Tech 49:73-83.

Dauber W, Voigt T, Heini A. 1999. Junctions between subsynaptic folds and rough sarcoplasmic reticulum of muscle fibres. J Muscle Res Cell Motil 20:697-701.

Dauber W, Voigt T, Härtel X, Meyer J. 2000. The T-tubular network and its triads in the sole plate sarcoplasm of the motor end-plate of mammals. J Muscle Res Cell Motil 21:443-449.

Decker ER, Dani JA. 1990. Calcium permeability of the nicotinic acetylcholine receptor: the single-channel calcium influx is significant. J Neurosci 10:3413-3420.

Dulhunty AF, Carter G, Hinrichsen C. 1984. The membrane capacity of mammalian skeletal muscle fibres. J Muscle Res Cell Motil 5:315-332.

Eisenberg BR, Kuda AM, Peter JB. 1974. Stereological analysis of mammalian skeletal muscle. I. Soleus muscle of the adult guinea pig. J Cell Biol 60:732-754.

Jasmin BJ, Cartaud J, Bornens M, Changeux JP. 1989. Golgi apparatus in chick skeletal muscle: changes in its distribution during end plate development and after denervation. Proc Natl Acad Sci U S A 86:7218-7222.

Jasmin BJ, Changeux JP, Cartaud J. 1990. Compartmentalization of cold-stable and acetylated microtubules in the subsynaptic domain of chick skeletal muscle fibre. Nature 344:673-675.

Jasmin BJ, Antony C, Changeux JP, Cartaud J. 1995. Nerve-dependent plasticity of the Golgi complex in skeletal muscle fibres: compartmentalization within the subneural sarcoplasm. Eur J Neurosci $7: 470-479$.

Kneissler U. 1997. Transmissions-Elektronenmikroskopie Teil2: Pioloform BM 18 zur Optimierung der physikochemischen Eigenschaften von Trägerfolien. MTA 12:322-324.

Peachey LD. 1965. The sarcoplasmic reticulum and transverse tubules of the frog's sartorius. J Cell Biol 25:209-231.

Ralston E. 1993. Changes in architecture of the Golgi complex and other subcellular organelles during myogenesis. J Cell Biol 120: 399-409.

Rambourg A, Segretain D. 1980. Three-dimensional electron microscopy of mitochondria and endoplasmic reticulum in the red muscle fiber of the rat diaphragm. Anat Rec 197:33-48.

Segretain D. 1995. Three-dimensional structure of the sarcoplasmic reticulum at the neuromuscular junctions of the rat diaphragm. Acta Anat Basel 154:224-231.

Tassin AM, Paintrand M, Berger EG, Bornens M. 1985. The Golgi apparatus remains associated with microtubule organizing centers during myogenesis. J Cell Biol 101:630-638.

Villa A, Podini P, Nori A, Panzeri MC, Martini A, Meldolesi J, Volpe P. 1993. The endoplasmic reticulum-sarcoplasmic reticulum connection. II. Postnatal differentiation of the sarcoplasmic reticulum in skeletal muscle fibers. Exp Cell Res 209:140-148.

Voigt T, Dauber W, Bensemann-Ryvkin I, Härtel X. 2002. On increasing membrane contrast by means of imidazole-osmium post-fixation as exemplified by skeletal muscle fiber. Microsc Res Tech 58:121124

Volpe P, Villa A, Podini P, Martini A, Nori A, Panzeri MC, Meldolesi J. 1992. The endoplasmic reticulum-sarcoplasmic reticulum connection: distribution of endoplasmic reticulum markers in the sarcoplasmic reticulum of skeletal muscle fibers. Proc Natl Acad Sci USA 89:6142-6146. 